\title{
A Geometric Morphometric Analysis of Heterochrony in the Cranium of Chimpanzees and Bonobos
}

\section{Citation}

Lieberman, Daniel E., Julian Carlo, Marcia Ponce de Leon, and Christoph P.E. Zollikofer. 2007. A geometric morphometric analysis of heterochrony in the cranium of chimpanzees and bonobos. Journal of Human Evolution 52(6): 647-662.

\section{Published Version}

doi:10.1016/j.jhevol.2006.12.005

\section{Permanent link}

http://nrs.harvard.edu/urn-3:HUL.InstRepos:3743586

\section{Terms of Use}

This article was downloaded from Harvard University's DASH repository, and is made available under the terms and conditions applicable to Other Posted Material, as set forth at http:// nrs.harvard.edu/urn-3:HUL.InstRepos:dash.current.terms-of-use\#LAA

\section{Share Your Story}

The Harvard community has made this article openly available.

Please share how this access benefits you. Submit a story.

\section{Accessibility}




\title{
A geometric morphometric analysis of heterochrony in the cranium of chimpanzees and bonobos
}

\author{
Daniel E. Lieberman ${ }^{\mathrm{a}, *}$, Julian Carlo ${ }^{\mathrm{b}}$, \\ Marcia Ponce de León ${ }^{c}$, Christoph P.E. Zollikofer ${ }^{c}$ \\ a Peabody Museum, Harvard University, Cambridge MA, 02138, USA \\ ${ }^{\mathrm{b}}$ Harvard Medical School, 220 Longwood Ave, Boston MA, 02115, USA \\ ${ }^{\mathrm{c}}$ Anthropologisches Institut, Universität Zürich-Irchel, Winterthurerstrasse 190, CH-8057 Zürich, Switzerland
}

Received 14 April 2006; accepted 19 December 2006

\begin{abstract}
Despite several decades of research, there remains a lack of consensus on the extent to which bonobos are paedomorphic (juvenilized) chimpanzees in terms of cranial morphology. This study reexamines the issue by comparing the ontogeny of cranial shape in cross-sectional samples of bonobos (Pan paniscus) and chimpanzees (Pan troglodytes) using both internal and external 3D landmarks digitized from CT scans. Geometric morphometric methods were used to quantify shape and size; dental-maturation criteria were used to estimate relative dental age. Heterochrony was evaluated using combined size-shape (allometry) and shape-age relationships for the entire cranium, the face, and the braincase. These analyses indicate that the bonobo skull is paedomorphic relative to the chimpanzee for the first principal component of size-related shape variation, most likely via a mechanism of postformation (paedomorphosis due to initial shape underdevelopment). However, the results also indicate that not all aspects of shape differences between the two species, particularly in the face, can be attributed to heterochronic transformation and that additional developmental differences must also have occurred during their evolution.
\end{abstract}

(C) 2007 Elsevier Ltd. All rights reserved.

Keywords: Heterochrony; Bonobo; Chimpanzee; Skull; Geometric morphometrics

\section{Introduction}

This study uses geometric morphometric (GM) methods to consider the extent to which the skull of the bonobo (Pan paniscus) is paedomorphic (juvenilized) compared to that of the chimpanzee (Pan troglodytes). A second, related goal is to test for any dissociation of heterochronies in the skull between facial and neurocranial regions in these two species. Applying heterochrony models to analyze differences between the bonobo and chimpanzee has not only been the subject of much

\footnotetext{
* Corresponding author.

E-mail addresses: danlieb@fas.harvard.edu (D.E. Lieberman), julian_carlo@hms.harvard.edu (J. Carlo), Marcia@ifi.unizh.ch (M. Ponce de León), zolli@aim.unizh.ch (C.P.E. Zollikofer).
}

previous research but also a major test case for theoretical debates concerning heterochrony theory (Shea, 1983a; 1984, 1992; Godfrey and Sutherland, 1996; Alba, 2002; Cobb and O'Higgins, 2004; Mitteroecker et al., 2004, 2005; Ponce de León and Zollikofer, 2006). Yet, despite this attention, bonobo and chimpanzee skulls differ morphologically in ways that have yet to be completely described or understood in terms of their developmental causes and evolutionary implications. While some authors (e.g., Shea, 1992) support the hypothesis that bonobos are paedomorphic relative to chimpanzees, others do not completely support this heterochronic diagnosis for several reasons. Godfrey and Sutherland (1996) used a multivariate allometric model to suggest that the bonobo cranium cannot be described as either paedomorphic or peramorphic relative to chimpanzees because of nonuniform allometries 
between the two species (see below). Additional studies by Williams et al. (2002), Mitteroecker et al. (2005), and Ponce de León and Zollikofer (2006) found that allometric scaling and multivariate analyses can explain only partially the differences between bonobo and chimpanzee crania. Mitteroecker et al. (2004, 2005), moreover, have raised questions about the utility of multivariate analyses such as GM to infer heterochronic transformations.

Here, we expand in several ways upon these earlier studies by using a landmark-based GM approach to compare ontogenetic changes in cranial shape and size between bonobos and chimpanzees using both external and internal landmarks, many of which represent key sites of craniofacial growth. We also use approximate estimates of age based on dental stages in the two species, and we examine multivariate size-shape and age-shape relationships throughout the cranium as a whole and within various regions. Given the potentially confusing nature of research on heterochrony, particularly in light of different methods and terminologies, we begin with a brief review of the GM heterochronic transformation model used here. We then review previous research on whether the bonobo skull is paedomorphic relative to the chimpanzee skull, and outline the hypotheses to be tested and the methods we employ.

\section{Heterochrony model}

Ever since Gould (1977), there has been considerable interest in heterochrony, the analysis of changes in the timing and/ or rate of developmental events among ancestor and descendant individuals or taxa. Traditionally, heterochronic processes are quantified with respect to three parameters: shape, size, and time. Size and shape describe the form of an organism, with size representing a scalar measure of the magnitude of form, and shape referring to aspects of form independent of scale (O'Higgins, 2000). Time can be quantified in several ways, typically as absolute age (chronological time relative to a homologous event such as birth), or in relative terms using a series of stages or events during ontogeny such as tooth eruptions (de Beer, 1958; Smith, 2001).

Implementation of the basic parameters of heterochronic transformation has varied considerably among studies, sometimes leading to alternative methodologies and terminologies (e.g., Gould, 1977; Alberch et al., 1979; McKinney and McNamara, 1991). A major source of confusion and debate has been the issue of time, for which reliable data are often unavailable in many samples. In the absence of good data on the ages of individuals in a sample, researchers typically use size as a proxy to compare size-shape relationships between ontogenetic samples of two or more taxa, effectively converting the analysis to a comparative ontogenetic allometry (Gould, 1977; Fleagle, 1985; McKinney and McNamara, 1991; Godfrey and Sutherland, 1996). Heterochrony and allometry provide complementary but not necessarily equivalent analytic frameworks for analyzing the evolution of ontogenetic trajectories (Klingenberg, 1998). Allometry tests for size-related shape changes; it cannot test directly for temporal differences in development, such as changes in rate and time of offset.
Although there exists a general covariance between size and ontogenetic time, the correspondence between allometry and heterochrony can be complex, making it difficult to infer heterochronic transformation from allometry. Alternative (and often confusing) terminologies have stemmed from multiple models that apply the same terms to heterochronic and allometric plots, which use different parameters to define the ontogenetic changes they portray. Allometry nonetheless sheds light on heterochronic transformations between two species when there is a strong covariance between size and age because heterochronic processes can create various patterns of scaling (McKinney and McNamara 1991; Godfrey and Sutherland, 1995a, b; Godfrey et al., 1998; Leigh et al., 2003).

Allometry, the relationship between size and shape, is used here explicitly as part of our analysis of heterochronic transformation. Changes in allometric trajectories indicate heterochronic transformation, but reflect only indirectly the effects of ontogenetic time (McKinney and McNamara 1991; Godfrey and Sutherland, 1995a, b; Klingenberg 1998; Smith, 2001; Leigh et al., 2003). The evolution of both allometric growth trajectories and aspects of developmental timing are therefore crucial for the understanding of heterochronic transformations because changes in the relationship between size and shape may be independent of that between shape and ontogenetic development.

Size and shape have also been treated in multiple ways. Until recently, most heterochrony studies assessed size using linear measurements, mass, areas, volumes, or occasionally the first principal component of variation of a set of linear measurements (for review, see Alba, 2002). Shape, a dimensionless parameter, has most often been quantified using ratios of two linear measurements. One problem with these measures of size and shape is the issue of size-shape covariation. Lengths, areas, and volumes are not always independent of shape, and the first principal component of multivariate analyses that are not computed from size-corrected data typically includes some component of size-related shape change (Jungers et al., 1995; Mitteroecker et al., 2004).

Geometric morphometric methods that use landmark data are a useful way to measure shape in heterochrony analyses because they can effectively quantify size and 3D shape independently (Zelditch et al., 1995; Penin et al., 2002, Berge and Penin 2004; Cobb and O'Higgins, 2004; Zollikofer and Ponce de León, 2004; Mitteroecker et al., 2004, 2005). Geometric morphometric methods first quantify the form (size and shape) of each specimen according to the location in space of a set of anatomical landmarks that are homologous among individuals. Shape and size are then separated using a Procrustes superimposition of landmarks, which translates the landmarks to a common origin, scales them to a common size, and rotates them to minimize their summed squared landmark distances (Rohlf and Slice, 1990; Zelditch et al., 2004). Procrustes superimposition thus enables one to quantify shape as the multidimensional deviation of a specimen's landmarks from a reference configuration, typically an average of the entire sample. Shape covariation is then quantified using principal components analysis (PCA), a dimension reduction technique 
that rotates the landmark configurations (using their covariance matrix) in multidimensional space to identify independent (uncorrelated) linear combinations of geometric landmark shifts (warps) (for discussion, see O'Higgins, 2000; Frost et al., 2003; Zelditch et al., 2004). Plotting the principal component (e.g., PC1 vs. PC2) scores of each individual therefore illustrates aspects of shape differences that covary within a particular shape space (O'Higgins, 2000; Eble, 2002). Note that some of the calculated principal components of shape may still be correlated with size, particularly those aspects of shape that covary with ontogeny. Such correlations permit multivariate allometries to be quantified by plotting them versus size. Size can be calculated in a number of ways, including centroid size, the square root of the sum of the squared distances among the landmarks in a configuration and their center of mass (Bookstein, 1991).

Although GM computations of shape present some complications when applied to heterochrony (see Discussion), this study applies GM measures of size and shape to a modification of Alberch et al.'s (1979) model of heterochrony (itself, a formalization of Gould, 1977) to compare the ontogenetic trajectories of the bonobo and chimpanzee. Alberch et al.'s (1979) model allows direct comparison of the dynamics of growth according to the parameters of shape, size, and time. While Alberch et al. (1979) plotted size, shape, and age on three axes, we reduce the inclusive $3 \mathrm{D}$ analysis to analyses of allometry (size vs. shape) and heterochronic transformation (age vs. shape) to simplify the depiction and description of ontogenetic differences (Shea, 1983, 1984, 1985). The heterochrony portion plots shape (expressed as principal components of variation among a set of landmarks) against age, allowing one to discern ontogenetic differences in the rate and timing of development by perturbations in initial development, slope, and termination of development (Klingenberg, 1998).

Figure 1 illustrates the diagnostic model employed here (but with several important assumptions that must be considered explicitly) in which differences in ontogeny between closely related species are divided into two categories of endpoint patterns: paedomorphosis and peramorphosis. In general, paedomorphosis refers to an adult descendant that resembles a juvenile ancestor (an underdeveloped descendant), and peramorphosis refers to an adult descendant that transcends an adult ancestor (an overdeveloped descendant). Paedomorphosis and peramorphosis can be further divided into several categories (representing different heterochronic processes) that are largely discernable using allometry, but which can differ in terms of the relationship between age and shape (Gould, 1977; see also Rice, 1997; Alba, 2002). One key problem to note, however, is that there are multiple trajectories of development that arrive at the same endpoint terminus between age and shape. For example, two species may arrive at the same adult relationship between shape and age, but one species may do so via growth spurts, while the other may have a constant rate of change between the two parameters. Here, for simplicity (and because of the nature of the data we analyze that compares two closely related and similar species) we assume that the regression between age and shape is linear in both species and varies either in slope or intercept. However, it is important to recognize that there is no reason to assume such linearity, and Fig. 1 does not include many alternative relationships between age and shape that differ in slope and intercept (the reader is encouraged to imagine a variety of nonlinear regressions). These caveats notwithstanding, paedomorphosis can occur according to the simplified model in Fig. 1 via at least five different processes based on the combination of the size-shape allometry and the endpoint termini of graphs of age vs. shape: (1) progenesis (also known as time hypomorphosis), in which the descendant prematurely ceases development but size and shape are not dissociated; (2) neoteny, in which the descendant has a reduced slope of allometry (size and shape are dissociated) without change in size; (3) rate hypomorphosis, in which size-shape relationships remain the same but a reduction in the rate of age-shape development leads to a reduction in size; (4) postformation, in which size-shape relationships remain the same but paedomorphosis occurs due to initial shape underdevelopment; and (5) postdisplacement, in which size-shape relationships remain the same but paedomorphosis is due to later onset of development. The respective peramorphic equivalents of these are hypermorphosis, acceleration, rate hypermorphosis, preformation, and predisplacement. Note that it is possible for a descendant to differ from its ancestor by more than one of the above processes.

Despite their advantages, multivariate measures of shape also have some drawbacks and potential complications when used to test hypotheses of heterochronic transformation. For one, the high dimensionality and resulting complexity of shape space can complicate or obscure the interpretation of ontogenies (Klingenberg, 1998; Tissot, 1988). Mitteroecker et al. (2004, 2005) have also pointed out that PCs derived from GM analyses are statistical constructions that combine growth from different regions and thus do not necessarily correspond to actual biological factors. According to their view, heterochronic transformations between two species can only be diagnosed if both "share the same ontogenetic trajectory in multivariate shape space" (Mitteroecker et al., 2005: 256). In other words, two species must have identical shape transformations for multiple PCs to be heterochronic variants. These authors found (using GM methods) that bonobos and chimpanzees share a similar ontogenetic trajectory for just PC1 and PC2, but not PC1 and PC3; hence, they rejected the hypothesis that heterochrony accounts for the differences between bonobos and chimpanzees. A further problem is that principal components analyses can be statistically unstable, so that slight changes to the data set (e.g. landmarks, samples) can change the PCs, resulting in the divergence of the trajectories.

Mitteroecker et al. (2005) raised some important and interesting points, but there are several reasons to reconsider their concerns about the use of individual PCs to test hypotheses of heterochronic transformation in comparisons of very similar taxa such as bonobos and chimpanzees. First, PCs derived from multivariate methods such as GM are no less biologically real as measurements of shape than simpler measurements such as the linear distance between two cranial landmarks 
PAEDOMORPHOSIS

(derived adult resembles juvenile ancestor)

Hypomorphosis (progenesis)
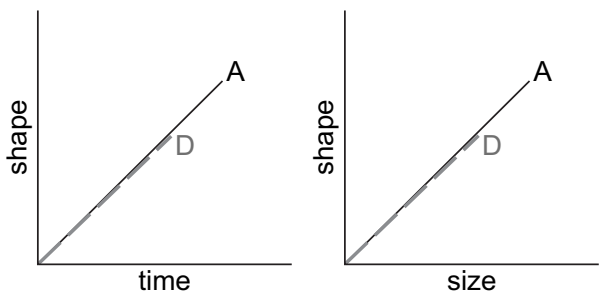

Neoteny
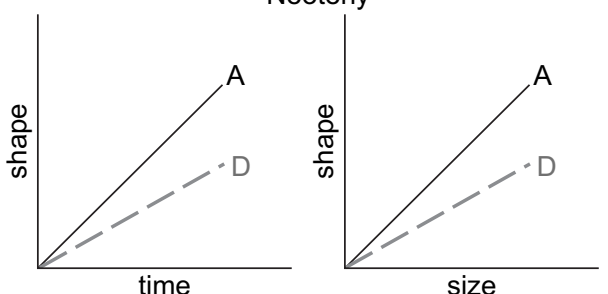

Rate hypomorphosis
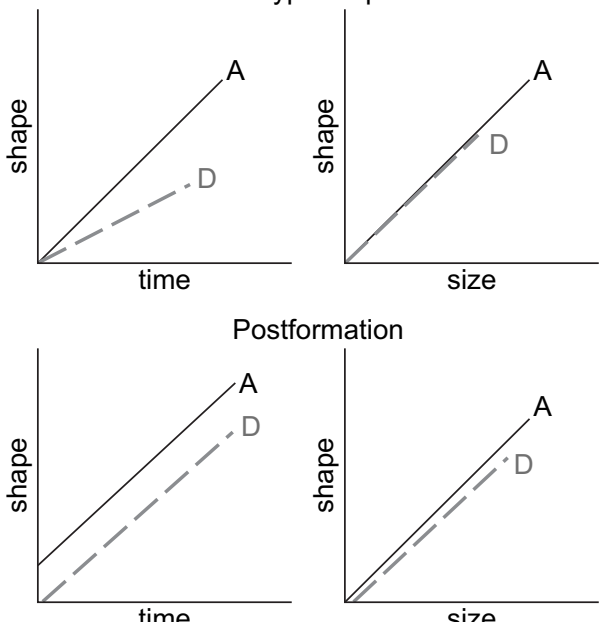

stformation
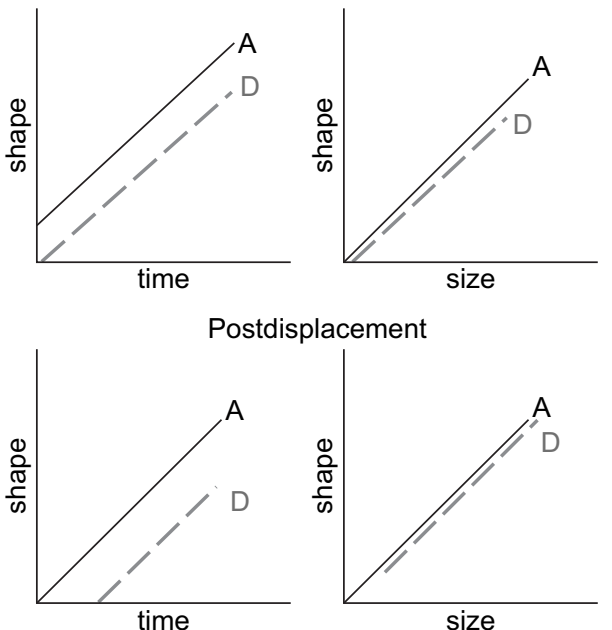

PERAMORPHOSIS

(derived adult transcends juvenile ancestor)

Hypermorphosis
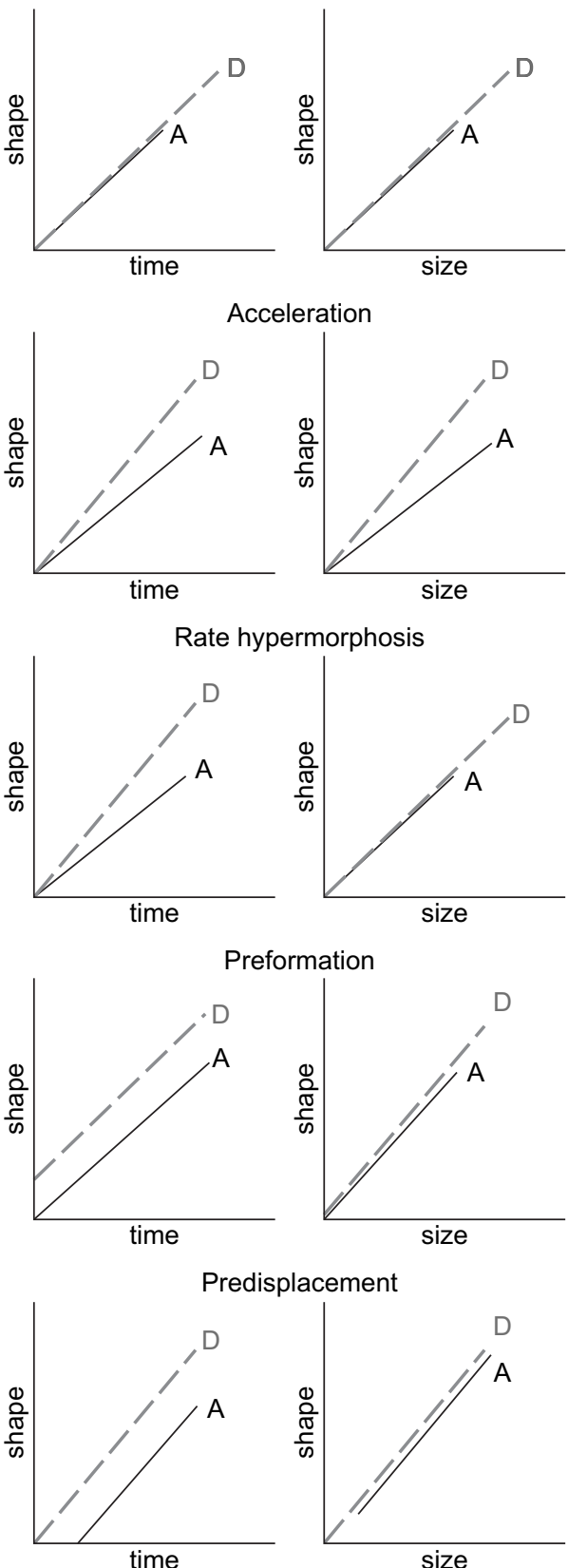

Fig. 1. Simplified heterochrony model used in this analysis (after Alba, 2002) that combines analyses of relative age vs. shape and size vs. shape. Note that multiple trajectories of development, slopes, and intercepts can produce the same endpoint terminus between age and shape. Here, we plot the slope between age and shape as linear in both species, with variation solely in either slope or intercept. The assumption of linearity, however, is not necessary and a more complete formulation of the model would require many alternative relationships between age and shape that differ in slope and intercept.

(or the ratio of two such measurements). For example, most univariate measurements- such as the chord from bregma to lambda - are integrated consequences of many developmental processes. In the case of bregma-lambda, these processes can include various effects of brain size expansion on sutural growth; many interactions between neural, basicranial, facial, and neurocranial growth processes; the effects of systemic hormones such as growth hormone; and so on (Weidenreich,
1941; Moss, 1958; Enlow, 1990; Lieberman, 1996; Lieberman et al., 2000a). Analyses of skulls typically find high degrees of distributed morphological covariation among most measurements because of myriad developmental processes that generate integration within and between regions. Viewed in this light, PCs may actually be better measures of shape than traditional caliper-based measurements because they are statistically independent, and derived without a priori models based 
on limited preconceptions about how skulls grow. In addition, PCs can and should be analyzed to derive testable hypotheses about particular developmental processes that generate covariation (Tissot, 1988). Software packages that convert shape spaces into visual models are particularly useful in this regard (for review, see Zelditch et al., 2004). Finally, PC1 by definition accounts for the largest proportion of variation in a data set. In comparing two slightly divergent trajectories among closely related species, it is biologically reasonable to infer that PC1 mostly captures their common direction of ontogeny through shape space, and the variance proportion (eigenvalue) of PC1 indicates their degree of commonality (O'Higgins, 2000). An even more complete, composite description of a common ontogenetic trajectory could be calculated via a multiple regression of all the PCs against size and/or time. The latter method, however, would compute an averaged similarity of transformation, based on multiple independent PCs, and would represent a composite of different, independent transformations. The utility of such a method for testing hypotheses of heterochrony is thus debatable.

A major concern of Mitteroecker et al. (2005) is the diagnosis of heterochrony for species that are not completely scaled variants of each other in multiple PCs (i.e., in some multivariate shape space). Their comparison of bonobos and chimpanzees included only plots of cross-sectional samples for the first three principal components of variation, but they did not plot the relationship of any PC to size or age. They discounted heterochrony as a mechanism of change between bonobos and chimpanzees because the two species had divergent trajectories of shape for PC2 and PC3 (see also Cobb and O'Higgins, 2004). In other words, they found that crosssectional sample of bonobos and chimps fell along the same trajectory for PC1 and PC2, but differed in terms of PC1 vs. PC3. A conservative way to interpret this divergence is to restrict diagnoses of heterochrony solely to PCs that are parallel in multivariate space, but this criterion is too stringent for several reasons. First, if two taxa had parallel PC trajectories in multiple dimensions, then they would have to be identical in their pattern of shape change, and hence distinguishable using primarily data on age or size. Two different species never exhibit exactly identical morphologies (and are thus identical in multivariate shape space) at different developmental stages. Requiring such colinearity is a sort of all-or-nothing criterion that is not what most biologists, including Gould (1977), have in mind when formulating hypotheses of heterochrony. Evolution is typically mosaic, generating change through heterochronic transformations of components-but not necessarily all aspects - of a descendent organism relative to its ancestor. For example, it is reasonable to hypothesize that the differences between bonobos and chimpanzees could arise through a heterochronic transformation of just facial or neurocranial growth, or through a different transformation of the face relative to the neurocranium. Principal components that show similar ontogenetic trajectories in two closely related species are likely to reflect ancestral patterns of scaling (see Zollikofer and Ponce de León, 2004), but multivariate analyses will almost always identify some PCs with divergent trajectories.
Species such as bonobos and chimpanzees may differ in part from multiple heterochronic transformations, as well as from other kinds of developmental shifts such as heterotopy (evolutionary change in spatial patterning). In this respect, principal components calculated from GM analyses are especially useful because they can help give an idea about what percentage of variation in a sample is accounted for by a particular shared pattern of covariation that scales with size and shape, and which is statistically independent of other patterns of covariation (Klingenberg, 1998). Thus, if PC1 scales with size in the same way for two species within the same shape space, then it is not problematic from the perspective of heterochrony for just $P C l$ that it may scale differently relative to other independent PCs, because each PC, by definition, quantifies different, independent patterns of shape covariation. That said, one must keep in mind that the percentage of variance explained by a given PC is also a function of nonbiological factors such as sample size, the number and type of landmarks included in the analysis, and so on.

Put differently, PCs calculated from GM analyses are usually graphed as bivariate regressions, so that it is often the case that two species may share the same ontogenetic trajectory (slope and, sometimes, intercept) for PC1 vs. PC2, but not for PC1 vs. PC3 and so on. Under what conditions can it be argued that any of these PCs represents a heterochronic transformation? We argue that if the combined shape space of the analysis is conservative (that is, changing a few variables or specimens does not alter the PCs substantially), and if a given PC (by definition, an independent measure of covariation) scales with size, then one can regress each PC separately against size and time to test hypotheses about allometry and heterochrony. Note that the first condition is more likely to be true for closely related species (such as bonobos and chimpanzees). In addition, the first PC of an analysis will usually correlate strongly with size, but additional information may be contained in other PCs for which correlation with size is not significant.

A final advantage of using GM analyses to analyze heterochrony even when not all PCs scale with allometry is that heterochronic processes can generate complex allometric patterns that may be difficult to interpret solely on the basis of a priori hypothesis testing (Mitteroecker et al., 2004). For example, it is possible that a given heterochronic transformation between an ancestral and descendant species may be regionally localized, such as in the face or in the neurocranium. However, many processes of development can also generate patterns of covariation that scale with size between regions. Such allometries may vary in terms of their pattern of ontogeny, and thus are amenable to heterochronic analysis.

\section{The bonobo hypothesis}

Ever since its discovery to science in 1929 , the bonobo has been suggested to be a dwarfed version of the common chimpanzee (Schwarz, 1929; Coolidge, 1933), with lower levels of sexual dimorphism in the skull and a smaller overall skull, 
particularly the face (Heintz, 1966; Cramer, 1977). The hypothesis that the bonobo is paedomorphic relative to the chimpanzee largely derives from the work of Shea (1983a), whose allometric comparisons showed that the adult bonobo was not simply a dwarfed version of the chimpanzee but instead a mosaic of differently proportioned skull, trunk and forelimb, and hindlimb regions. Since total skull length relative to headfork length had a lower slope of allometry in the bonobo than the chimpanzee, Shea (1983a) proposed that the bonobo was paedomorphic through the process of neoteny.

As often pointed out (e.g., Godfrey and Sutherland, 1996; Klingenberg, 1998; Alba, 2002), Shea's (1983a) initial analysis had several limitations in relation to the question of whether the bonobo skull is paedomorphic. First, total skull length is not a measurement of skull shape. Second, skull length or other measurements of overall skull shape may confound the existence of multiple, dissociated heterochronic shifts, given the partial modular independence of the face and the complex formed by the basicranium and neurocranium (Moss and Young, 1960; Cheverud, 1982, 1995; Lieberman et al., 2000a, b; Hallgrimsson et al., 2007). Third, Shea's initial analysis included no data on age, which are necessary to diagnose some forms of paedomorphosis. Finally, because both chimpanzee and bonobo skulls scale with negative allometry to body length, the lower slope revealed by Shea's analysis would mean that the bonobo skull is actually peramorphic, despite its smaller size (Godfrey and Sutherland, 1996). ${ }^{1}$ This does not mean, however, that bonobos actually have peramorphic skulls because diagnoses of heterochronic growth processes are valid only insofar as they are undertaken within a single growth region rather than between different growth regions that may scale differently (Alba, 2002).

Since Shea's (1983a) study, others have made further allometric comparisons of bonobo and chimpanzee cranial growth, and these have varied in their methods and findings. These studies differ from that of Shea (1983a) by describing shape as ratios of interlandmark distances and using size measurements that are intrinsic to the skull. When interlandmark distances are compared, mainly using basicranial length and palate length to represent the growth regions of the neurocranium and face, respectively, there is strong evidence for ontogenetic scaling between these measurements (Shea 1983b, 1984, 1985, 1992), implying that the bonobo is paedomorphic through hypomorphosis (or progenesis). When shape is defined as the ratio between basicranial length and palate length, and basicranial length is used as a proxy for size, then the bonobo is reduced in size and juvenalized in shape compared to the chimpanzee at the end of development, but size and shape are not dissociated from each other (Alba, 2002). More indepth studies (e.g., Shea, 1984, 2000), which compared a larger number of cranial interlandmark distances, found the majority of comparisons to scale ontogenetically (i.e., with the same coefficient of allometry). Interestingly, those few comparisons

\footnotetext{
${ }^{1}$ Shea's (1983a) analysis mistakenly reversed the labels for bonobos and chimpanzees in his Fig. 1C; when corrected, they lead to a diagnosis of peramorphosis (see Shea, 2000).
}

revealing dissociated allometries placed the bonobo on the peramorphic side of dissociation from the common allometry: the cranium is wider and the infratemporal fossa and zygomatic root are larger in bonobos relative to basicranial length.

Recent landmark-based GM studies have improved our understanding of the morphological relationship between the bonobo and chimpanzee within multivariate shape space, confirming earlier findings that they deviate from pure ontogenetic scaling. Williams et al. (2002) found that multivariate scaling only partially accounts for differences between bonobos and chimpanzees. In a GM study of facial landmarks, Cobb and O'Higgins (2004) found that, although the bonobo and chimpanzee appear to have a common ontogenetic shape trajectory compared to the gorilla, when PC1 and PC2 are plotted against each other (together, explaining 54\% of the shape variation), the trajectories of other PCs are significantly divergent. In addition, Mitteroecker et al.'s $(2004,2005)$ analysis of the first three principal components of craniofacial landmarks among chimpanzees and bonobos confirmed that the two species follow different ontogenetic shape trajectories after birth for PCs other than PC1. In other words, some of the differences between bonobo and chimpanzee skulls appear to be a consequence of developmental changes other than heterochronic transformation, including heterotopy-the rotation or translocation of growth fields relative to one another that leads to divergent ontogenetic trajectories in shape space (Zelditch and Fink, 1996).

Although the GM analyses of Cobb and O'Higgins (2004) and Mitteroecker et al. $(2004,2005)$ revealed potential nonheterochronic differences in development between bonobos and chimpanzees, these studies did not test hypotheses of heterochronic transformation directly or explicitly, in part because they did not incorporate data on age. In addition, these studies did not test separately for regional heterochronies in the skulls, such as in the face and in the neurocranium, which comprise two different potential modules. This analysis therefore builds on previous studies by addressing the question of bonobo paedomorphosis using GM methods to measure multivariate shape and size both within the skull as a whole and within the two major modular units of the skull, the face and the neurobasicranial complex (NBC) (Lieberman et al., 2000a). In addition, we attempted to estimate relative age using dental-maturation criteria, allowing a full analysis of heterochrony.

Two major hypotheses are tested. Hypothesis 1 is that the bonobo skull as a whole is paedomorphic with respect to the chimpanzee. Hypothesis 2 is that any heterochronic transformations of the facial and neurocranial regions between these species are decoupled from one another, and that only part of the bonobo skull is paedomorphic with respect to the chimpanzee. For simplicity, both hypotheses assume that the bonobo is the descendant species, but we caution that this assumption is neither necessarily true nor testable given available data. As outlined in Klingenberg (1998) and Alba (2002), the two hypotheses are tested using data on the parameters of size, shape, and relative age (stage). Allometry is used to test for a common trajectory of size vs. shape, against which deviations from scaling can be determined. 


\section{Materials and methods}

\section{Samples}

This study used CT scans of 30 Pan troglodytes and 19 Pan paniscus skulls, which are listed in Table 1 by sex and dental stage. Although this reduces sample sizes, CT scans are used in order to include major landmarks of growth that are on the inside of the cranium and that cannot be measured from external landmarks (see below). Use of CT-scanned skulls also allows us to estimate age using crown and root development (see below). The chimpanzee skulls come primarily from a single population of Liberian chimpanzees from the collections of the Peabody Museum of Harvard University; these were supplemented by four juveniles from the A.H. Schultz collection (Anthropological Institute and Museum, University of Zurich, Switzerland). Chimpanzees from the Peabody Museum were scanned using a Siemens multiarray-detector scanner with a slice thickness of $0.5 \mathrm{~mm}$ (reformatted into isotropic voxels of $0.488 \mathrm{~mm}$ ); chimpanzees from Zurich were scanned using a Siemens Somatom Sensation multislice CT scanner with a slice thickness of $0.5 \mathrm{~mm}$, and serial cross sections reconstructed at $0.4-\mathrm{mm}$ intervals. The bonobos are from the collection of the Musée de l'Afrique Centrale in Tervuren, Belgium. All but four were scanned by J. Braga using a Siemens Somatom Esprit Spiral CT (slice thickness typically varied among samples but ranged between 0.33 and 0.50 ). Four additional adult bonobos from Tervuren were included in the sample (29040, 29045, 23509, 26939); these individuals were scanned at the University Hospital, Leuven (Belgium), using a Siemens Somatom Sensation CT scanner with a slice thickness of $0.5 \mathrm{~mm}$. Note that both samples comprised multiple individuals of both sexes from all stages of development-infant, juvenile, subadult, and adult - as assessed by dental stage (see below). One limitation of the sample is its modest size, which results from restricting the sample to CT-scanned crania. Restricting the analysis to scanned individuals is useful in order to incorporate internal landmarks that provide information on the location of key growth sites (such as in the cranial base) that are unavailable from external landmarking methods; in addition, the sample is large enough to estimate confidence limits for slopes and intercepts using standard statistical methods.

\section{Aging}

Age is the most challenging parameter to estimate for the samples used here, particularly for bonobos, for which there are almost no developmental data from known-age samples (for reviews, see Kinzey, 1984; Smith et al., 1994). Several challenges need to be considered in order to incorporate age estimates in this study. The most problematic issue is circularity of logic. Given the absence of aging studies on bonobos, it is necessary to use studies of chimpanzees and other apes to estimate bonobo ages. Although it is not possible to use comparative data to estimate the chronological age of the bonobos sampled here, several lines of evidence suggest that it is reasonable to use dental-maturation criteria from other African
Table 1

Sample used

\begin{tabular}{|c|c|c|c|}
\hline Individual & Species & Dental age $^{1}$ & Sex \\
\hline 26938 & P. paniscus & 3.75 & M \\
\hline 29021 & P. paniscus & 4.5 & $?$ \\
\hline 29022 & P. paniscus & 5.5 & $?$ \\
\hline 29029 & P. paniscus & 6.5 & $?$ \\
\hline 26990 & P. paniscus & 2.75 & F \\
\hline 26979 & P. paniscus & 3.5 & M \\
\hline 26958 & P. paniscus & 3.5 & M \\
\hline 26992 & P. paniscus & 2.25 & $\mathrm{~F}$ \\
\hline 29008 & P. paniscus & 1.5 & $\mathrm{~F}$ \\
\hline 22336 & P. paniscus & 3.75 & $?$ \\
\hline 26977 & P. paniscus & 3.5 & $\mathrm{~F}$ \\
\hline 29061 & P. paniscus & 2.75 & M \\
\hline 27010 & P. paniscus & 5.75 & $\mathrm{~F}$ \\
\hline 29066 & P. paniscus & 9 & M \\
\hline 29052 & P. paniscus & $11+$ & $?$ \\
\hline 29040 & P. paniscus & $11+$ & $\mathrm{F}$ \\
\hline 29045 & P. paniscus & $11+$ & $\mathrm{F}$ \\
\hline 23509 & P. paniscus & 10.5 & M \\
\hline 26939 & P. paniscus & $11+$ & M \\
\hline AM6892 & P. troglodytes & 3.25 & $\mathrm{~F}$ \\
\hline AM7659 & P. troglodytes & 1.25 & F \\
\hline AM8606 & P. troglodytes & 2.5 & M \\
\hline AS1813 & P. troglodytes & 2.5 & M \\
\hline 6918 & P. troglodytes & 10.5 & $\mathrm{~F}$ \\
\hline 7288 & P. troglodytes & 7.5 & $\mathrm{~F}$ \\
\hline 7286 & P. troglodytes & 10.5 & $\mathrm{~F}$ \\
\hline 6917 & P. troglodytes & $11+$ & M \\
\hline 7591 & P. troglodytes & 7.25 & M \\
\hline 7562 & P. troglodytes & $11+$ & $\mathrm{F}$ \\
\hline 6915 & P. troglodytes & $11+$ & M \\
\hline 6913 & P. troglodytes & $11+$ & M \\
\hline 7296 & P. troglodytes & 5 & M \\
\hline 7270 & P. troglodytes & 8.5 & $\mathrm{~F}$ \\
\hline 7266 & P. troglodytes & 2.5 & $\mathrm{~F}$ \\
\hline 7542 & P. troglodytes & 9 & M \\
\hline 7566 & P. troglodytes & $11+$ & M \\
\hline 6923 & P. troglodytes & $11+$ & M \\
\hline 6929 & P. troglodytes & 8.5 & M \\
\hline 7560 & P. troglodytes & 8.5 & $\mathrm{M}$ \\
\hline 7545 & P. troglodytes & $11+$ & $\mathrm{F}$ \\
\hline 6953 & P. troglodytes & $11+$ & M \\
\hline 7280 & P. troglodytes & 3 & $\mathrm{~F}$ \\
\hline 7283 & P. troglodytes & $11+$ & M \\
\hline 6961 & P. troglodytes & 9 & $\mathrm{~F}$ \\
\hline 6949 & P. troglodytes & $11+$ & $\mathrm{F}$ \\
\hline 6970 & P. troglodytes & $11+$ & $\mathrm{M}$ \\
\hline 7262 & P. troglodytes & 6 & M \\
\hline 7265 & P. troglodytes & $11+$ & $\mathrm{F}$ \\
\hline 7284 & P. troglodytes & 6.75 & $\mathrm{~F}$ \\
\hline
\end{tabular}

Individuals that had reached full dental maturity are listed as $11+$.

${ }^{1}$ Relative age estimates based on Kuykendall (1996) and Dean and Wood (1981) (see text for details).

apes to estimate their relative ontogenetic stage, which is a useful variable to employ in heterochrony analyses (for review, see Smith, 2001). First, although the sequence of tooth eruption is notoriously variable within species, the general eruption sequences in bonobos and chimpanzees are essentially identical for both the permanent and deciduous dentitions (Boughner and Dean, 2004), with delay of the I 2 being the most common difference (Kinzey, 1984). Second, estimates of the age of 
eruption of deciduous teeth from a small sample of bonobos $(n=4)$ fall within the chimpanzee range, although at the low end (Smith et al., 1994). Finally, there is general similarity in the postnatal timing of tooth eruption and the relationship of these events to craniofacial maturation among the great apes. As Fig. 2 shows, the ages of eruption of the permanent teeth are not significantly different in captive male and female chimpanzees, and in a pooled sample of captive gorillas. Given these similarities, as well as those of various fossil apes (e.g., Beynon et al., 1998; Kelley and Smith, 2003), it is reasonable to assume that the sequence and relative timing of dental development in bonobos and chimps are sufficiently similar to compare them using the same criteria. Put differently, it would be surprising if the general sequence, pattern, and relative rate of eruption of the dentition in bonobos differed significantly from that of other African apes. Finally, even if the chronological rate of dental developmental differs between these species, the relationship between these events and craniofacial development appears to be broadly conserved among primates. Most importantly, M1 eruption occurs near the time of the cessation of neural growth, and M3 eruption occurs close to the completion of craniofacial growth (Schultz, 1960; Smith, 1989, 1991; Kelley and Smith, 2003).

A second problem to consider is that recent research (Zihlman et al., 2004) has confirmed that wild animals mature more slowly, but in the same sequence, than captive animals, for which almost all aging data come (see Fig. 2). Therefore, more longitudinal data from wild populations of all ape species would be preferable in order to estimate their absolute ages. In actual fact, rate differences between wild and captive populations highlight the point made above that dental-aging estimates for all the species analyzed here must be treated as relative rather than absolute age estimates. Such estimates have their limitations but are nonetheless fully compatible with the logic of heterochrony analysis (Gould, 1977, Smith, 2001; German, 2004).

A third problem is that the only aging data available for the genus Pan come from cross-sectional studies (Dean and Wood, 1981; Kuykendall, 1996; Anemone et al., 1996), which yield more error than longitudinal studies. Longitudinal data from both species would be better (but present their own problems in terms of aligning individual trajectories), but as shown by German (2004), cross-sectional data are still useful despite their lower resolution when there is general similarity in terms of the slope of the relationship between ontogenetic events and time. For the time being, lack of longitudinal data limits the resolution of all studies of heterochrony in apes.

Accordingly, ontogenetic stage was estimated for both species using two studies: one by Dean and Wood (1981) and the other by Kuykendall (1996). First, CT images of each mandible were used to estimate age using tooth-developmental stages from Dean and Wood's (1981) study. Because Kuykendall

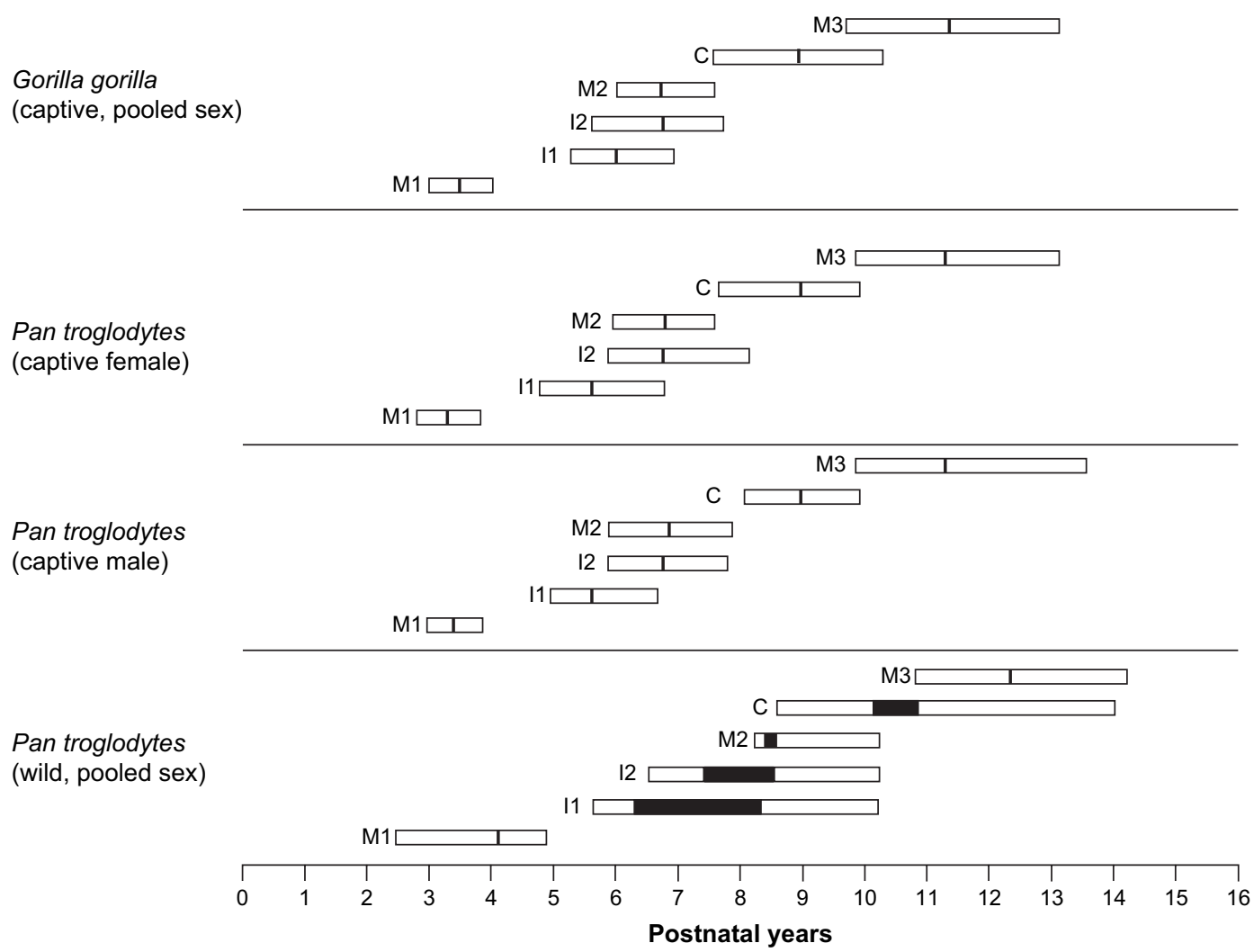

Fig. 2. Age of eruption of the permanent dentition of African apes, captive vs. wild, based on data from Smith et al. (1994) and Zihlman et al. (2004). Solid bars indicate means, including error ranges for mean estimates from Zihlman et al. (2004). Note that, although wild chimpanzees are shifted toward more delayed eruption times, the general pattern and sequence of eruption timings do not vary between species. 
(1996) showed that only canines displayed sexual dimorphism in tooth eruption in Pan, canine maturation was not considered in the analysis. As a second step, estimates based on Dean and Wood's (1981) stages were then checked against age estimates derived using the tooth-formation stages for $\mathrm{I}_{1}, \mathrm{M}_{1}, \mathrm{M}_{2}$, and $\mathrm{M}_{3}$ from Kuykendall's (1996) study. In all cases, the dental-age estimate derived using Dean and Wood's (1981) stages fell within the range derived from Kuykendall's (1996) stages. Note that the ontogenetic-age estimates used here are not estimates of absolute chronological age for either species. In other words, the heterochronic transformations that can be diagnosed in this analysis are limited to changes in the relative sequence of ontogenetic events, not absolute time.

\section{Landmarks}

Twenty-seven craniofacial landmarks, listed and defined in Table 2, were used. The landmarks were chosen to represent points from the neurocranium, face, and cranial base, and they include a number of internal sites of craniofacial growth. According to Bookstein's (1991) categorization, 30\% are type I landmarks (points where three structures meet or sites of locally unusual histology); 60\% are type II landmarks (points whose homology is supported by geometric criteria, such as tooth tip or maxima of curvature); and only $10 \%$ are type III landmarks (extremal points whose definitions refer to information from diverse, finitely separated locations such as endpoints). After rendering the skulls using VG StudioMax Pro ${ }^{\circ}$, each landmark was expressed as a point in 3D space using Cartesian coordinates. To assure correct placement, all landmarks were taken by examining the location of the point (indicated visually by cross-hairs) on both the rendered cranium and in three orthogonal planes (sagittal, coronal, and transverse). In the rare case of a missing landmark due to specimen damage, the landmark was estimated as accurately as possible using visual means from the surrounding skeletal tissue.

Landmark precision and accuracy were both evaluated. Precision was evaluated by taking all 27 craniofacial landmarks from one chimpanzee (\#6918) on five separate occasions. Two-tailed paired Student's $t$-tests between pairs of landmark-coordinate sets found no significant differences $(p>0.61)$. The average standard deviation of landmark coordinates in the $x, y$, and $z$ planes was between 0.50 and $0.57 \mathrm{~mm}$, indicating that landmark locations can be determined consistently and with an acceptable degree of error. Accuracy was evaluated by comparing interlandmark distances obtained through VG StudioMax Pro ${ }^{\circledR}$ with distances obtained using electronic calipers on the same cranium. Nine interlandmark distances were used: nasion-rhinion, bregma-lambda, zygomaxillary orbitale-zygofrontal orbitale, basion-incisive canal, basion-palatal intersection point, nasion-metopion, upper orbital rim-zygomaxillary orbitale, jugale-C-I2 alveolar ridge, and basion-zygomaxillare. A two-tailed paired $t$ test of the distance values found no significant difference $(p=0.87)$ between these two sets with an average interlandmark difference of $0.52 \mathrm{~mm}$.

\section{Geometric morphometrics}

Morphologika $^{\odot}$ software Version 2.0 (www.york.ac.uk/res/ fme/resources/software.htm) was used to translate, rotate, and then scale via a generalized least squares (GLS) superimposition the landmark configurations for all individuals; the program was then used to calculate and graph the principal components of shape variation using the variance-covariance matrix of the resulting Procrustes residuals (O'Higgins, 2000). Size was calculated as centroid size (i.e., the square root of the sum of the squared deviations of landmarks from a centroid point). These analyses were performed not only on the skull as a whole, but also separately on subsets of landmark points from the face using landmarks $1-15$ and from the NBC (neurocranium and basicranium) using landmarks 1627. Shape changes in the skull were visualized using a wireframe connecting adjoining landmarks (Fig. 3).

Best-fit reduced major axis (RMA) regression lines for chimpanzees and bonobos were calculated, and differences in the slopes and intercepts were tested using ANCOVA (calculated from least square regressions) with an alpha level of 0.05 to indicate significance. In order to avoid assuming linearity, significance was also evaluated using ANOVA comparisons of the two species divided into four age categories: I, up to 3 years; II, from 3 to 6 years; III, from 6 to 9 years; IV, greater than 9 years. To facilitate interpretation of the bivariate scattergrams, individuals in Figs. $4-7$ are differentiated by sex and the four age categories.

\section{Results}

\section{Principal components of shape variation}

Analysis of all 27 cranial landmarks resulted in 42 nonzero eigenvectors, whose eigenvalues indicate a pattern of shape variation that is primarily quantified by PC1 (71.7\%) (Table 3). Permutations of the analysis that alter the number of variables and individuals result in little change to the shape space (data not shown). Figure 4A, which plots the first two PCs, indicates that PC1 discriminates to some extent between the two species, but with overlap. The bonobo and chimpanzee samples create clouds that reflect trajectories of development from negative to positive PC1 scores (left to right). When adult males are removed from the analysis, an ANCOVA finds no significant difference in the slope of PC1 vs. PC2 for either species. Wireframes of the shape space quantified by the PCs indicate that each component quantifies an integrated suite of features that reflects several coordinated ontogenetic changes that have also been documented in other analyses of ape ontogeny (Mitteroecker et al., 2004, 2005; Cobb and O'Higgins, 2004; Guy et al., 2005). Low values for PC1 represent individuals with smaller, shorter palates; shorter faces that are more ventrally rotated under the cranial vault; rounder and relatively larger cranial vaults; and more flexed cranial bases. Individuals with more positive PC1 values have relatively larger and more prognathic faces that are more dorsally rotated, have a relatively smaller and more oval-shaped 
Table 2

Landmarks used

\begin{tabular}{|c|c|c|c|}
\hline Landmark & Type $^{1}$ & Region & Definition \\
\hline 1 Nasion & I & Face & Midline intersection of nasal and frontal bones \\
\hline 2 Glabella & II & Face & Most anterior midline point on the frontal bone at the level of the supraorbital torus \\
\hline 3 Rhinion & II & Face & Midline point at the inferior end of the internasal suture \\
\hline 4 Anterior nasal spine & II & Face & Most anterior and posterior point on nasal spine \\
\hline 5 Incisive canal & II & Face & Most anterior and inferior midline point of incisive canal on the hard palate \\
\hline 6 Palatal intersection point & I & Face & Midline intersection of palatal and palatine sutures \\
\hline 7 Zygomaxillary-orbitale & $\mathrm{I} / \mathrm{II}$ & Face & Point where zygomaxillary suture intersects orbital margin \\
\hline 8 Uppermost orbital rim & II & Face & Most superior point on the orbital margin \\
\hline 9 Ectochonchion-zygofrontal & $\mathrm{I} / \mathrm{II}$ & Face & Point where zygofrontal suture intersects orbital margin \\
\hline 10 Zygomaxillare & $\mathrm{I} / \mathrm{II}$ & Face & Most inferior and lateral point on the zygomaxillary suture \\
\hline 11 Alare & II & Face & Most lateral point on the margin of the nasal aperture \\
\hline 12 Maxillary tuberosity & II & Face & Most posterior point of the alveolar crest below the palatine suture \\
\hline 13 M1-M2 alveolar margin & II & Face & Point at center of alveolar crest between $\mathrm{M}^{1}$ and $\mathrm{M}^{2}$ \\
\hline $14 \mathrm{C}-\mathrm{I} 2$ alveolar margin & II & Face & Point at center of alveolar crest between $\mathrm{I}^{2}$ and the canine \\
\hline 15 Jugale & II & Face & Deepest point of notch between the temporal and frontal processes of the zygomatic \\
\hline 16 Basion & II & $\mathrm{NBC}^{2}$ & Most anterior and inferior midline point on the margin of the foramen magnum \\
\hline 17 Sella & III & NBC & Point (in space) at the center of the sella turcica \\
\hline 18 Foramen caecum & I & $\mathrm{NBC}$ & Most anterior inferior point of anterior cranial base \\
\hline 19 Sphenoidale & II & NBC & Most superior and posterior midline point on the tuberculum sellae \\
\hline 20 Posterior cribiform plate & II & NBC & Most posterior and inferior point on surface of cribiform plate \\
\hline 21 Spheno-occipital synchondrosis & II & NBC & Exterior midline point on the suture between the sphenoid and occipital \\
\hline 22 Parietopion & III & NBC & Midline ectocranial point at the greatest elevation above the chord from bregma to lambda \\
\hline 23 Opisthion & II & NBC & Midline point at posterior margin of the foramen magnum \\
\hline 24 Opisthocranion & III & NBC & Midline ectocranial point at the farthest chord length from the glabella \\
\hline 25 Lambda & I & $\mathrm{NBC}$ & Ectocranial midline point of actual or projected intersection of sagittal and lambdoidal sutures \\
\hline 26 Bregma & I & NBC & Ectocranial midline point at intersection of coronal and sagittal sutures \\
\hline 27 Metopion & III & $\mathrm{NBC}$ & $\begin{array}{l}\text { Ectocranial midline point on frontal at its greatest elevation relative to the chord from nasion } \\
\text { to bregma }\end{array}$ \\
\hline
\end{tabular}

1 Types correspond to categories of Bookstein (1991).

2 NBC, neurobasicranial complex (see Lieberman et al., 2000a).

neurocranium, and more extended cranial bases. The second PC, which accounts for only $5.5 \%$ of the variation, primarily separates individuals with higher versus lower cranial vaults, particularly above the orbits.

Plots of the first two principal components in analyses of just the facial landmarks (1-15) and just the NBC landmarks (16-27) reveal interesting differences, with much less variance explained by the first principal component. In the facial analysis (Fig. 4B), the first PC accounts for only $36.4 \%$ of the total variation, but discriminates the two species at all but the earliest ontogenetic stage. With adults removed, the slopes of the ontogenetic trajectories differ significantly for the two species (ANCOVA, $p<0.05$ ). Individuals with higher PC1 scores are more prognathic, have a longer rostrum, particularly in the posterior portion of the face, and have relatively smaller orbits. The second PC (12.1\%) primarily describes variations in palate angle and facial width. In the NBC landmark analysis (Fig. 4C), the PC1 axis discriminates the two species but with ontogenetically staggered overlap and no significant difference in slope between the two species. Bonobos generally have lower PC1 values than chimps of the same relative ontogenetic stage. The first $\mathrm{PC}$ in this analysis explains $56.7 \%$ of the variance (Table 3). As is evident from Fig. 4C, individuals with lower PC1 scores have more flexed cranial bases, with more globular braincases, more anteriorly positioned foramina magna, and relatively shorter anterior cranial bases. The second PC explains relatively less variation, primarily in terms of the relative height of landmarks at the posterior end of the cranial vault (many of which are type III landmarks). As in the face, the $\mathrm{PC} 1$ axis primarily discriminates bonobos and chimpanzees, with bonobos and

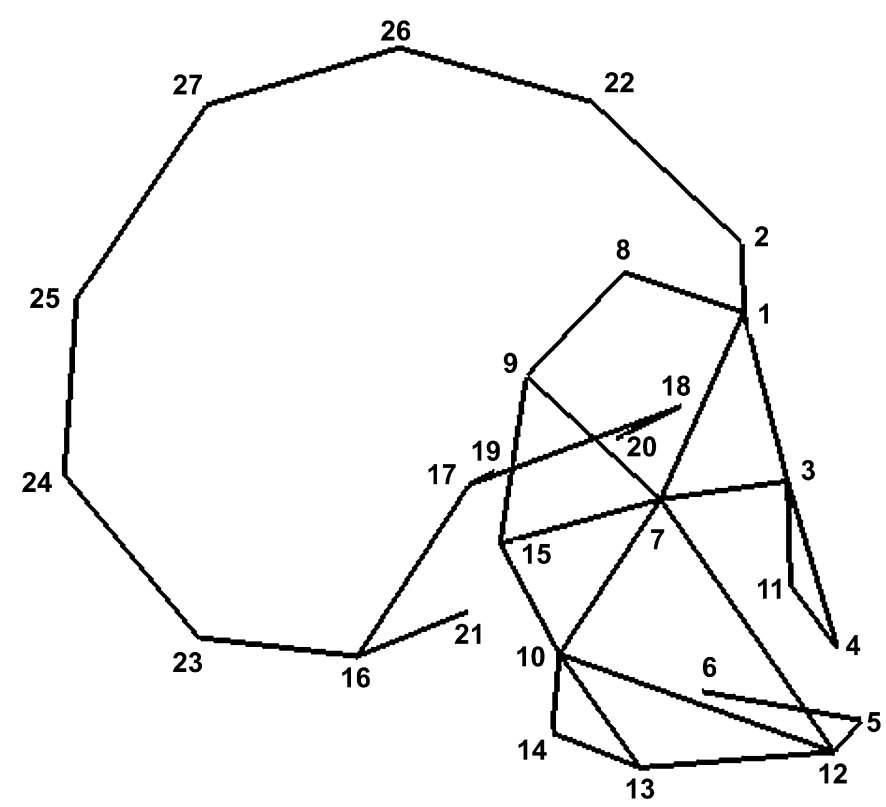

Fig. 3. Landmarks used in the analyses (numbers correspond to definitions in Table 2). 


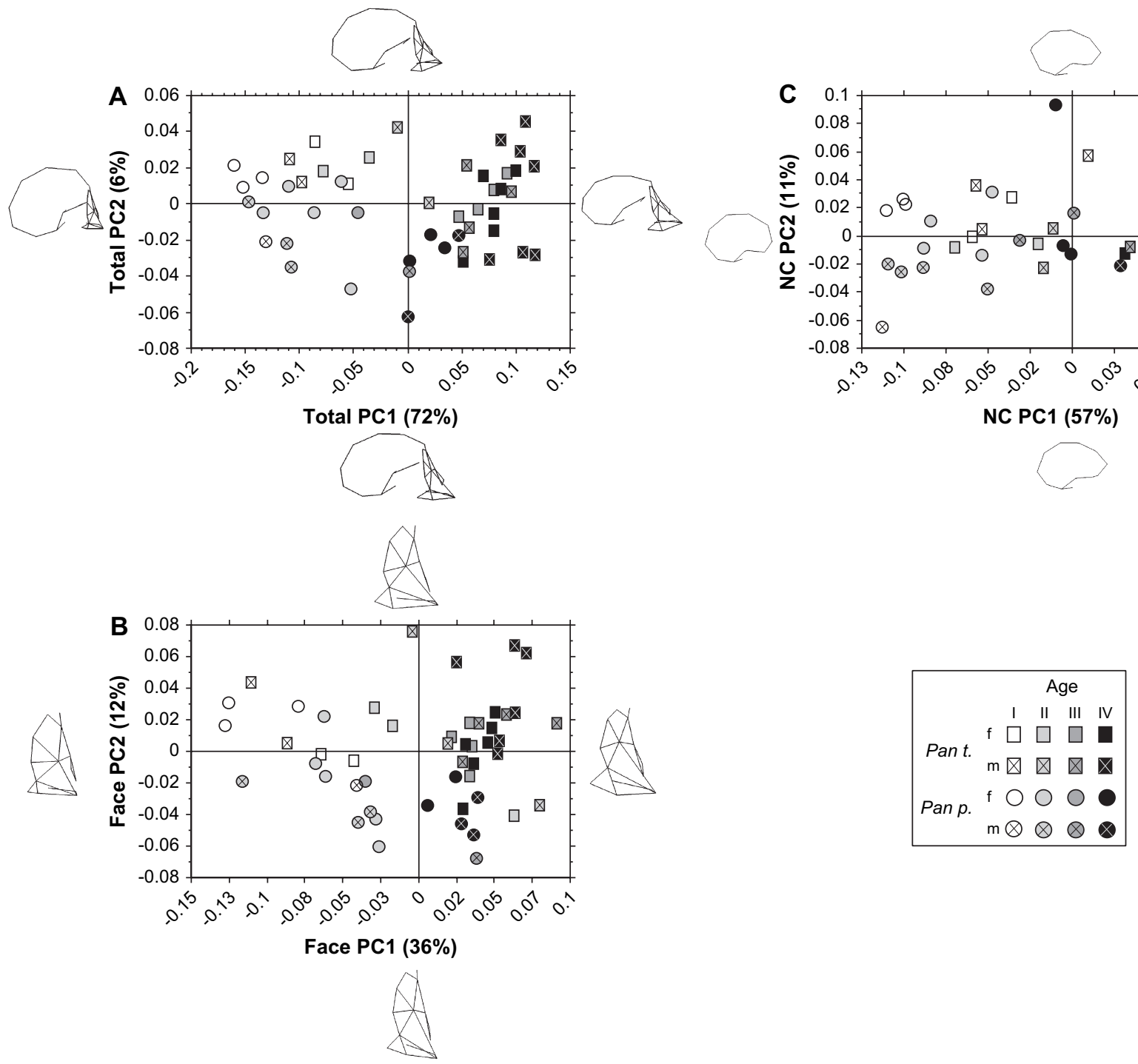

Fig. 4. Plots of PC1 vs. PC2 for analyses of the entire cranium (A), face (B), and neurocranium and basicranium (C). Wireframes indicate extreme landmark configurations for each principal component.

chimpanzees generally occupying respectively smaller and larger PC1 values. On this axis, adult bonobos lie in the same shape space as young chimpanzees. The second PC does little to discriminate individuals by sex, age, or species in the facial and NBC analyses.

\section{Heterochrony and allometry}

Rates of shape change in both species were quantified by graphing PCs for each landmark set against age estimates, and ontogenetic allometries were quantified using centroid size against PCs (which were previously calculated from size-normalized data as described above). Because significant correlations $(p<0.05)$ were only found between PC1 and either relative age or size, we focus solely on PC1 of each analysis. Figure 5 and Tables 4 and 5 summarize the results for the entire cranium. When graphed in terms of heterochrony (Fig. 5A) both species have parallel shape trajectories with significantly different intercepts but not significantly different slopes. Thus, for any given relative age, the bonobos are underdeveloped relative to chimpanzees, but fall along the same shape trajectory. These differences are confirmed by the ANOVA results (Table 5), which indicate that PC1 scores by age category are different at or near conventional levels of significance for all age categories, despite small sample sizes and the crudeness of the age categories themselves. However, the ontogenetic allometry (Fig. 5B) indicates that both species do not differ significantly in either slope or intercept when PC1 is plotted against centroid size. In combination, these results diagnose bonobos as paedomorphic relative to chimps via postformation, or initial underdevelopment for just this component of shape (i.e., just PC1). Note that the adult bonobos (male and female) fall in the shape space of the subadult chimpanzees for PC1.

Figure 6 plots analyses of just the facial landmarks. In terms of relative age versus shape (Fig. 6A), the intercept but not the slope of the RMA regression between PC1 of facial shape and dental age is significantly different (see Table 4) 

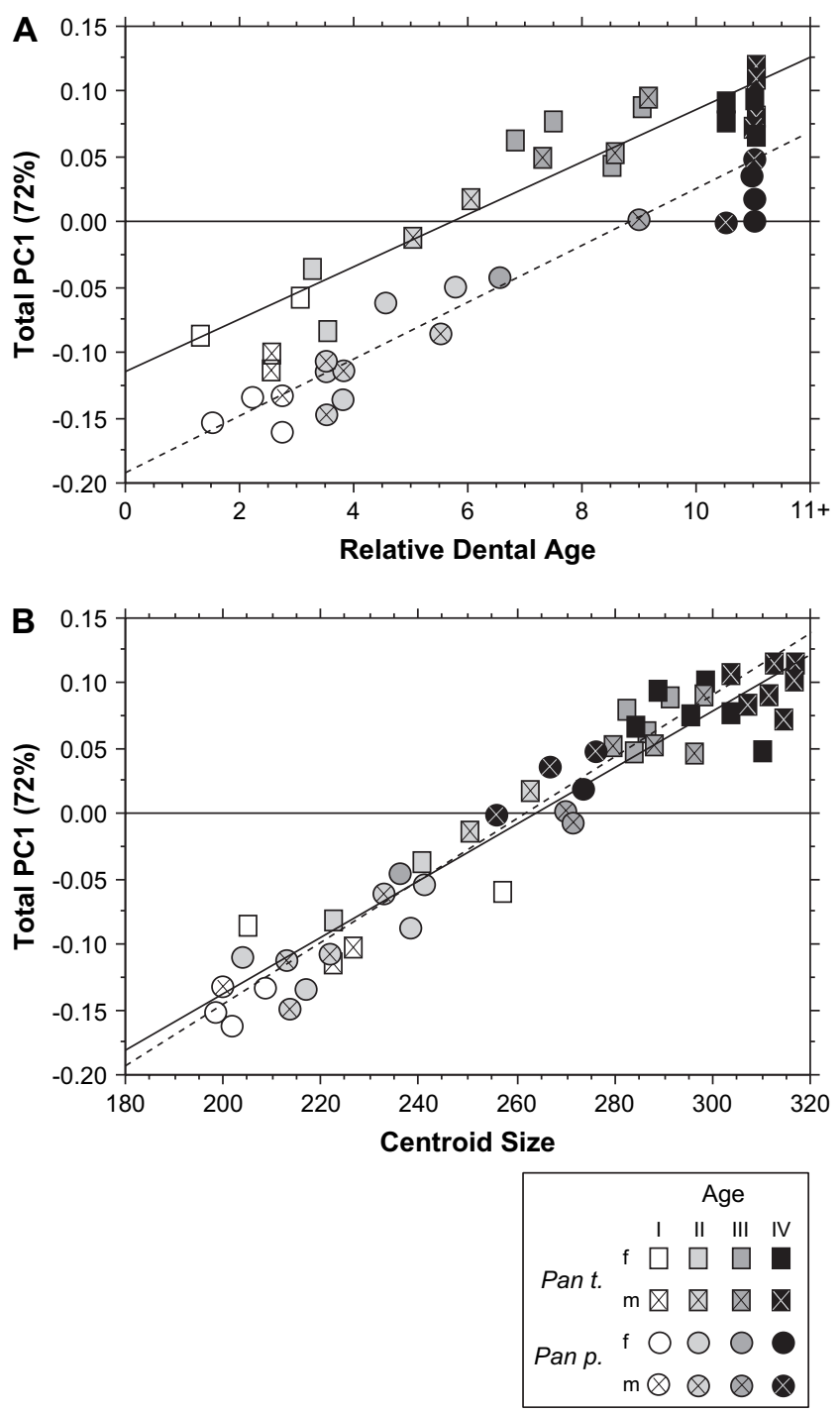

Fig. 5. PC1 vs. dental age (A) and centroid size (B) for entire landmark set.

between the two species. The same is also true, but to a lesser extent, of the ontogenetic allometry (Fig. 6B), and there is a general trend for bonobos of the same general stage to have smaller values for PC1 at all stages except the first (Table 5). As in the entire cranial analysis, the shape of the face for the largest adult bonobos falls in the shape space of subadult chimpanzee faces for PC1. To some extent, these results diagnose bonobo faces as paedomorphic relative to chimpanzees via postformation for just $P C l$, but much less strongly than in the analysis of the entire cranium. Therefore, one cannot conclude that the bonobo face is predominantly different from chimpanzees via paedomorphosis because PC1 explains less than $40 \%$ of the total variance of this particular sample. As always, larger samples of adult bonobos and juvenile chimps and more landmarks will help to clarify these results.

Finally, Fig. 7 and Tables 4 and 5 summarize results from analyses of just the landmarks from the neurocranium and basicranium (NBC). These analyses generally resemble those for the entire cranium, with significantly different intercepts but not slopes for PC1 vs. dental age, and no significant difference
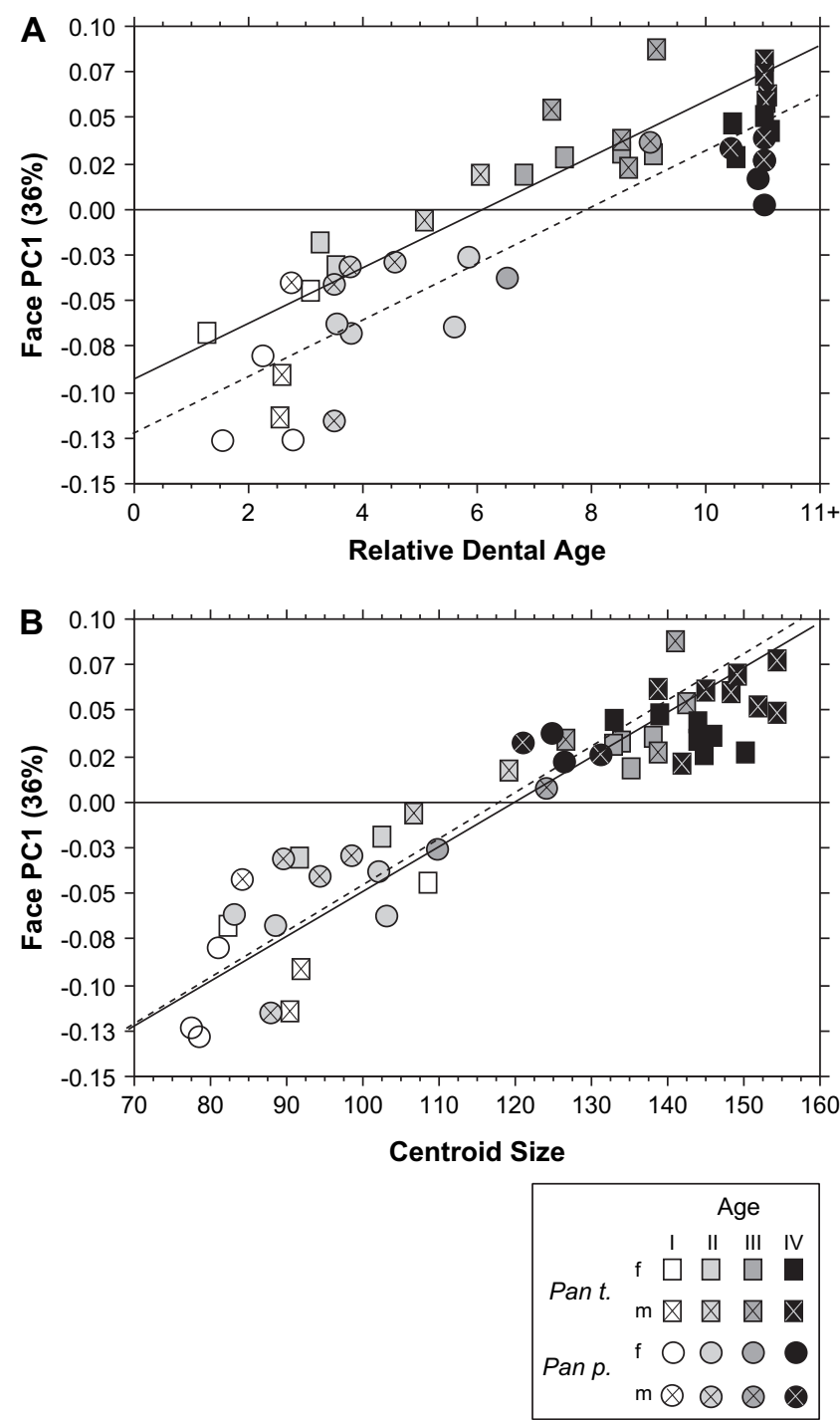

Fig. 6. PC1 vs. dental age (A) and centroid size (B) for facial landmark set.

in slope or intercept for PC1 vs. centroid size. As was the case with respect to the whole cranium, the bonobo NBC appears to be paedomorphic via postformation, with adult bonobos falling in the shape space of subadult juvenile chimpanzees for PC1 (56.7\% of the variance) and no statistically significant difference in ontogenetic shape trajectory.

\section{Discussion}

Overall, the results presented here lend support to the hypothesis that the bonobo cranium differs partially but not entirely from the chimpanzee cranium because of paedomorphosis. In particular, results for the entire cranium indicate paedomorphosis via postformation (initial shape underdevelopment) for just the first principal component of variation because analyses of $\mathrm{PC} 1$ versus estimates of relative age indicate similar slopes but with a higher intercept for the chimpanzee sample. In addition, the ontogenetic allometries of the two species for PC1 are the same in terms of slope and intercept, but with adult bonobos falling in the shape space of subadult 

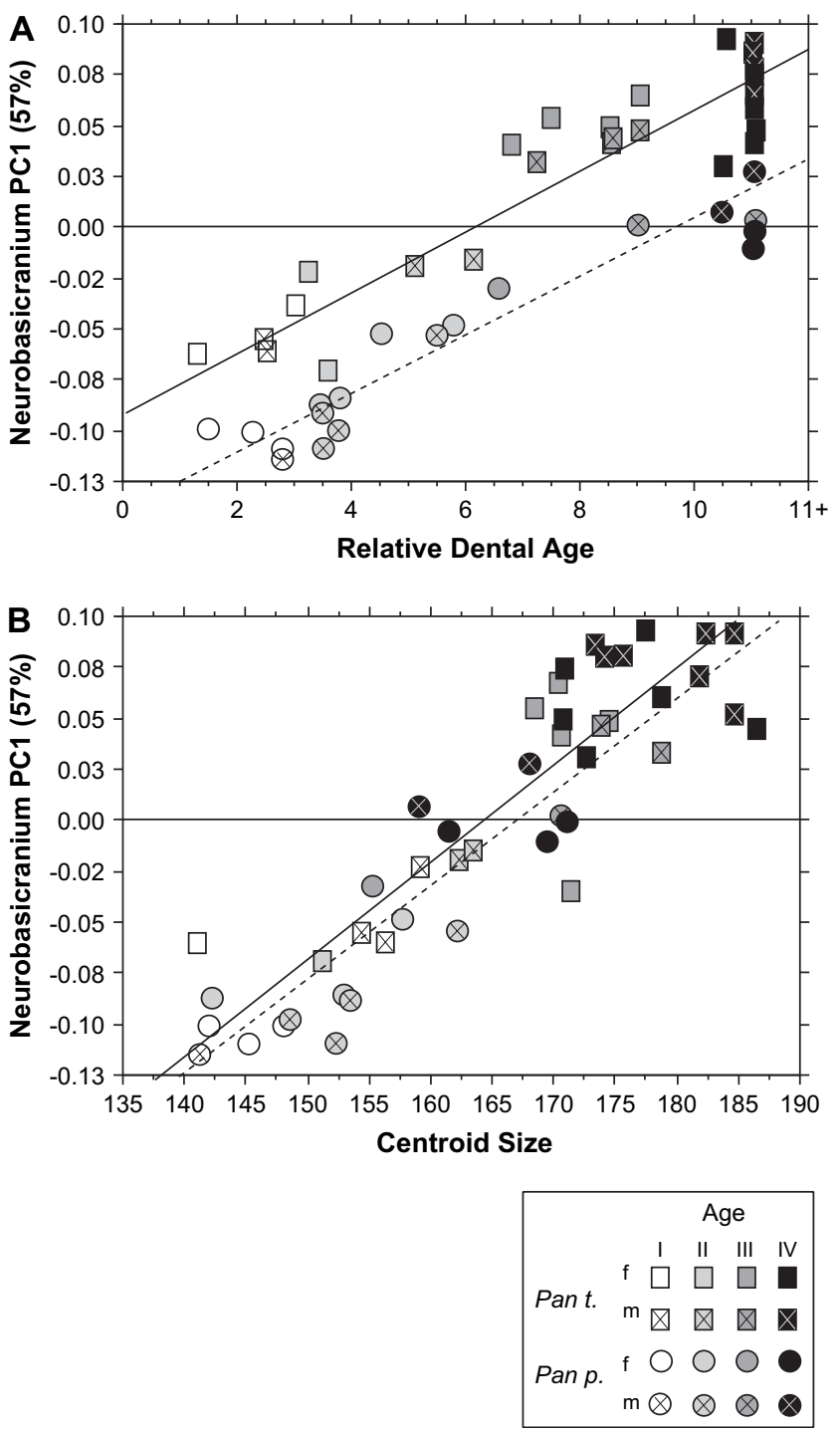

Fig. 7. PC1 vs. dental age (A) and centroid size (B) for neural and basicranial landmark set.

chimpanzees. Thus, bonobo and chimp cranial growth follow the same ontogenetic allometric trajectory for PC1, but for any given age, bonobo crania are underdeveloped relative to those of chimpanzees. These results generally support previous hypotheses of paedomorphosis for bonobos (e.g., Shea, 1983a, b, 1984, 1985; Alba, 2002; Ponce de León and Zollikofer, 2006), but differ slightly in the type of paedomorphosis diagnosed, and in the extent to which paedomorphosis is responsible for the species' contrasts.

As noted by other authors (e.g., Mitteroecker et al., 2005), it can be problematic to analyze the entire cranium as a whole. Importantly, the above results offer limited support for the second hypothesis that heterochronic transformations of the facial and neurocranial regions are partly dissociated from one another and thus contribute differently to paedomorphosis of the cranium as a whole. As noted above, the results for the NBC generally resemble those of the entire cranium, suggesting paedomorphosis via postformation, but the analysis of
Table 3

PCA variance components ( $>5 \%$ ) for whole skull, face, and vault

\begin{tabular}{lccc}
\hline & Cranium & Face & Neurobasicranium \\
\hline PC1 & 71.7 & 36.4 & 56.7 \\
PC2 & 5.5 & 12.1 & 10.9 \\
PC3 & - & 10.2 & 5.6 \\
PC4 & - & 7.2 & - \\
PC5 & - & 5.0 & - \\
\hline
\end{tabular}

only facial landmarks indicates significant differences in the intercept between age vs. PC1 and size vs. PC1. In other words, the face does not appear to be paedomorphic in the same pattern and to the same extent as the NBC or the entire cranium. Recall also that PC1 in the facial analysis only explains $38 \%$ of the total variance in this sample. This calculation does not mean that the bonobo face is precisely $38 \%$ paedomorphic because the exact proportion of variance explained by any given component is a function of many factors, including the size and nature of the sample, as well as the landmarks used. Further analyses with larger samples and better age data will help clarify the results reported here, but it is reasonable to conclude that other developmental differences such as heterotopy are likely to explain a large if not dominant proportion of the facial differences between these samples. Interestingly, similar results were obtained by Cobb and O'Higgins (2004) for just facial landmarks using a larger sample of hominoid species. Other researchers have also shown that the face is more paedomorphic relative to the NBC in bonobos compared to chimpanzees (Cramer, 1977; McHenry and Corruccini, 1981; Shea 1983a; Alba, 2002).

Regardless of what patterns of paedomorphosis explain the variation quantified by $\mathrm{PC} 1$, the results of this analysis also support the hypothesis that the bonobo skull is only partially paedomorphic with respect to the chimpanzee. More precisely, the bonobo is partially paedomorphic relative to the chimpanzee, more so in the vault and cranial base, and less so in the face. However, as discussed above, a problematic issue is determining what percentage of variation can be explained by paedomorphosis or any other pattern of heterochrony. Mitteroecker and colleagues (2005) argued that one cannot diagnose heterochrony using GM or other multivariate analyses if the first few principal components of variation do not follow identical ontogenetic trajectories. However, one advantage of using GM methods to quantify shape variation is that they identify combinations of variables that covary independently and that scale with size and age. Thus, the methods employed here are able to detect without a priori models what aspects of the bonobo cranium are heterochronic transformations of the chimpanzee cranium (or vice versa). Such diagnoses still need to be interpreted biologically using developmental data from other kinds of analyses. In fact, these diagnoses are primarily useful for generating hypotheses about developmental shifts (Lieberman et al., 2004).

Another way to consider this issue is via a thought experiment. If one agrees that evolutionary change can occur through multiple mechanisms including (but not exclusively) heterochrony, then how would one set about to generate and 
Table 4

Reduced major axis regression results ${ }^{1}$

\begin{tabular}{|c|c|c|c|c|c|c|c|c|}
\hline & \multicolumn{3}{|c|}{ Bonobo $(n=19)$} & \multicolumn{3}{|c|}{ Chimpanzee $(n=30)$} & \multicolumn{2}{|c|}{ ANCOVA } \\
\hline & Intercept $( \pm$ s.d. $)$ & Slope ( \pm s.d.) & $r$ & Intercept ( \pm s.d. $)$ & Slope ( \pm s.d.) & $r$ & Slope & Intercept \\
\hline \multicolumn{9}{|l|}{ Entire cranium } \\
\hline CS vs. PC1 & $-0.657(0.04)$ & $0.003(0.003)$ & 0.966 & $-0.568(0.04)$ & $0.002(0.0003)$ & 0.887 & NS & NS \\
\hline Age vs. PC1 & $-0.190(0.08)$ & $0.020(0.003)$ & 0.963 & $-0.127(0.01)$ & $0.013(0.002)$ & 0.869 & NS & $*$ \\
\hline \multicolumn{9}{|l|}{ Facial landmarks } \\
\hline CS vs. PC1 & $-0.487(0.05)$ & $0.002(0.001)$ & 0.867 & $-0.273(0.03)$ & $0.002(0.001)$ & 0.895 & NS & $*$ \\
\hline Age vs. PC1 & $-0.128(0.05)$ & $0.015(0.004)$ & 0.874 & $-0.097(0.01)$ & $0.015(0.003)$ & 0.869 & NS & $*$ \\
\hline \multicolumn{9}{|c|}{ Neurobasicranial landmarks } \\
\hline CS vs. PC1 & $-0.809(0.05)$ & $0.005(0.001)$ & 0.878 & $-0.778(0.09)$ & $0.005(0.002)$ & 0.829 & NS & NS \\
\hline Age vs. PC1 & $-0.137(0.04)$ & $0.014(0.002)$ & 0.953 & $-0.092(0.02)$ & $0.015(0.002)$ & 0.933 & NS & $*$ \\
\hline
\end{tabular}

${ }^{1}$ Abbreviations are as follows: CS, centroid size; NS, not significant; ${ }^{*} p<0.05$.

test hypotheses about what developmental shifts actually occurred in a particular evolutionary transformation? Until recently, researchers primarily addressed such questions using a typical hypotheticodeductive method, taking measurements of cross-sectional samples of two or more species, and analyzing the slope and intercept of various combinations of the parameters. Variables that scaled significantly with size were then analyzed further using various heterochrony models, whereas variables uncorrelated with size were discounted from further analysis. Such studies therefore incorporated the assumption (often implicit) that not all transformations between ancestral and descendant species are captured by allometry and heterochrony. However, the methods they used offered few means of quantifying how much of the total variation between the species was related to scaling, and for diagnosing other mechanisms that could cause nonallometric changes. Therefore, the logic used by Mitteroecker et al. (2005) applies no less to all kinds of heterochrony analyses; it is simply more obvious from GM analysis (a point they acknowledged). As they noted, GM methods offer advantages unavailable to simple univariate approaches because they provide estimates (albeit context-dependent) of the percentage of variation that particular transformations cause, they can help generate hypotheses about the biological mechanisms that might account for these transformations, and they may also diagnose alternative mechanisms to be explored.

Viewed in this light, the results reported here confirm those of other GM analyses relating to this problem. Heterochrony alone is an insufficient framework to interpret whatever evolutionary changes occurred to make bonobos and chimpanzees different from one another. In this analysis, PC1 accounts for a strikingly limited proportion $(<50 \%)$ of variation in the face, and the first PCs of the entire cranial and NBC analyses do not account for $25 \%$ and $33 \%$ of the variation, respectively. These data thus support the conclusions of other GM analyses (Cobb and O'Higgins, 2004; Mitteroecker et al., 2004, 2005; Ponce de León and Zollikofer, 2006) that scaling alone cannot explain all of the differences in cranial shape between bonobos and chimpanzees. Instead, future research using different analytical methods and additional data should focus on potential nonheterochronic contributions to the differences evident between the species. This conclusion is evident from differences in the ontogenetic trajectories between the species, and from the fact that other PCs that explain significant amounts of variance have no obvious or significant correlation with sex or age for these analyses (e.g., PC2 in Fig. 4). A related caveat to consider is that correlations in shape change expressed by principal components only quantify changes in the configuration of the landmarks included in the analysis. It follows that the biological processes that underlie these changes may not be adequately represented by any one principal component, and/or a single principal component may correspond to more than one covarying process of change (Tissot, 1988). Ultimately, the shape changes these principal components describe need to be related to particular developmental processes.

Finally, while some proportion of the differences between bonobo and chimpanzee crania apparently derive from paedomorphosis, additional data are needed to test more securely the diagnosis of paedomorphosis via postformation. Most crucially, we need new methods to age individuals without reference to cross-sectional samples of captive populations. However, it is reasonable to hypothesize that larger and better-aged samples will confirm the above results given the

Table 5

ANOVA results for relative dental age vs. PC 1 (standard error in parentheses)

\begin{tabular}{|c|c|c|c|c|c|c|c|c|c|}
\hline \multirow[t]{2}{*}{ Age } & \multicolumn{3}{|c|}{ Total cranium (PC1) } & \multicolumn{3}{|c|}{ Face (PC1) } & \multicolumn{3}{|c|}{ Neurobasicranium (PC1) } \\
\hline & Chimpanzee & Bonobo & $p$ & Chimpanzee & Bonobo & $p$ & Chimpanzee & Bonobo & $p$ \\
\hline $0-3.0$ & $0.09(0.01)$ & $0.15(0.01)$ & 0.06 & $-0.08(0.02)$ & $-0.09(0.02)$ & NS & $-0.05(0.01)$ & $-0.11(0.00)$ & 0.003 \\
\hline $3.1-6.0$ & $0.03(0.02)$ & $0.10(0.01)$ & 0.07 & $-0.01(0.01)$ & $-0.06(0.01)$ & 0.02 & $-0.03(0.01)$ & $-0.08(0.01)$ & 0.009 \\
\hline $6.1-9.0$ & $-0.06(0.01)$ & $0.02(0.01)$ & $<0.001$ & $0.04(0.01)$ & $0.00(0.01)$ & 0.08 & $0.05(0.00)$ & $-0.02(0.1)$ & $<0.001$ \\
\hline$>9.0$ & $-0.09(0.00)$ & $-0.02(0.01)$ & $<0.001$ & $0.05(0.00)$ & $0.02(0.01)$ & 0.07 & $0.07(0.00)$ & $0.00(0.01)$ & 0.001 \\
\hline
\end{tabular}


findings of previous analyses using external landmarks and/or measurements from much larger samples (e.g., Shea, 1983a, 1984). Regardless of these caveats, bonobos evidently not only end their ontogeny at a smaller size and more juvenile shape than chimpanzees, but they also begin postnatal development at smaller sizes and more juvenile shapes. Indeed, bonobos at birth are approximately $0.5 \mathrm{~kg}$ lighter than chimpanzees (Smith and Leigh, 1998). Developmental differences between these two species thus appear to be present before birth, presumably as the result of regulatory shifts in fetal or embryonic development. It is interesting to speculate that such mechanisms-perhaps differences in circulating hormone levels or hormone receptor binding activities-may link the correlated suite of shifts evident in postcranial anatomy, the brain, or other organs that play some role in the behavioral differences between the species (Shea, 1983a, 1984, 1989; Blount, 1990). Testing these hypotheses is of importance not only for understanding how and why bonobos and chimpanzees differ, but also for understanding better how patterns of developmental differences in the phenotype can help identify candidate mechanisms and even the genes responsible for evolutionary developmental differences between closely related species.

\section{Acknowledgements}

We are very grateful to J. Braga for generously providing CT scans of bonobo crania. We also thank J. Braga, S. Cobb, C. Dean, R. German, B. Hallgrimsson, S. Leigh, R. McCarthy, P. O’Higgins, D. Pilbeam, P. Ratiu, B. Shea, B.H. Smith, and R. Wrangham for discussions, comments, and technical assistance; several anonymous referees also provided thoughtful comments. Funding was provided in part by the American School of Prehistoric Research, the Department of Anthropology, Harvard University, and by the Swiss National Science Foundation (grant \#31-67209.01).

\section{References}

Alba, D.M., 2002. Shape and stage in heterochronic models. In: MinughPurvis, N., McNamara, K.J. (Eds.), Human Evolution through Developmental Change. Johns Hopkins University Press, Baltimore, pp. 28-50.

Alberch, P., Gould, S.J., Oster, G.F., Wake, D.B., 1979. Size and shape in ontogeny and phylogeny. Paleobiology 5, 296-317.

Anemone, R.L., Mooney, M.P., Siegel, M.I., 1996. Longitudinal study of dental development in chimpanzees of known chronological age: implications for understanding the age at death of Plio-Pleistocene hominids. Am. J. Phys. Anthropol. 99, 119-133.

Berge, C., Penin, X., 2004. Ontogenetic allometry, heterochrony, and interspecific differences in the skull of African apes, using tridimensional Procrustes analysis. Am. J. Phys. Anthropol. 124, 124-138.

Beynon, A.D., Dean, M.C., Leakey, M.G., Reid, D.J., Walker, A., 1998. Comparative dental development and microstructure of Proconsul teeth from Rusinga Island, Kenya. J. Hum. Evol. 35, 163-209.

Blount, B.G., 1990. Issues in bonobo (Pan paniscus) sexual behavior. Am. Anthropol. 92, 702-714.

Bookstein, F.L., 1991. Morphometric Tools for Landmark Data: Geometry and Biology. Cambridge University Press, Cambridge.
Boughner, J.C., Dean, M.C., 2004. Does space in the jaw influence the timing of molar crown initiation? A model using baboons (Papio anubis) and great apes (Pan troglodytes, Pan paniscus). J. Hum. Evol. 46, 255-277.

Cheverud, J.M., 1982. Phenotypic, genetic and environmental morphological integration in the cranium. Evolution 36, 499-512.

Cheverud, J.M., 1995. Morphological integration in the saddle-back tamarin (Saguinus fuscicollis) cranium. Am. Nat. 145, 63-89.

Cobb, S.N., O'Higgins, P., 2004. Hominins do not share a common postnatal facial ontogenetic shape trajectory. J. Exp. Zool. (Mol. Dev. Evol.) 302B, 302-321.

Coolidge, H.J., 1933. Pan paniscus: pygmy chimpanzee from south of the Congo River. Am. J. Phys. Anthropol. 18, 1-57.

Cramer, D.L., 1977. Craniofacial morphology of Pan paniscus: a morphometric and evolutionary appraisal. Contrib. Primatol. 10, 1-64.

Dean, M.C., Wood, B.A., 1981. Developing pongid dentition and its use for ageing individual crania in comparative cross-sectional growth studies. Folia Primatol. 36, 111-127.

de Beer, G.R., 1958. Embryos and Ancestors, third ed. Oxford University Press, Oxford.

Eble, G.J., 2002. Multivariate approaches to development and evolution. In: Minugh-Purvis, N., McNamara, K.J. (Eds.), Human Evolution through Developmental Change. Johns Hopkins University Press, Baltimore, pp. 51-78.

Enlow, D.H., 1990. Facial Growth, third ed. W.J. Saunders, Philadelphia.

Fleagle, J.G., 1985. Size and adaptation in primates. In: Jungers, W.L. (Ed.), Size and Scaling in Primate Biology. Plenum Press, New York, pp. 1-19.

Frost, S.R., Marcus, L.F., Bookstein, F.L., Reddy, D.P., Delson, E., 2003. Cranial allometry, phylogeography, and systematics of large-bodied papionins (Primates: Cercopithecinae) inferred from geometric morphometric analysis of landmark data. Anat. Rec. 275A, 1048-1072.

German, R.Z., 2004. The ontogeny of sexual dimorphism: the implications of longitudinal vs. cross-sectional data for studying heterochrony in mammals. In: Anapol, F., German, R.Z., Jablonski, N.G. (Eds.), Shaping Primate Evolution. Cambridge University Press, Cambridge, pp. 11-23.

Godfrey, L.R., Sutherland, M.R., 1995a. Flawed inference: why size-based tests of heterochronic processes do not work. J. Theoret. Biol. 172, 43-61.

Godfrey, L.R., Sutherland, M.R., 1995b. What's growth got to do with it? Pattern and process in the evolution of ontogeny. J. Hum. Evol. 29, 405-431.

Godfrey, L.R., Sutherland, M.R., 1996. The paradox of peramorphic paedomorphosis: heterochrony in human evolution. Am. J. Phys. Anthropol. 99, 17-42.

Godfrey, L.R., King, S.J., Sutherland, M.R., 1998. Heterochronic approaches to the study of locomotion. In: Strasser, E., Fleagle, J., Rosenberger, A., McHenry, H.M. (Eds.), Primate Locomotion: Recent Advances. Plenum Press, New York, pp. 277-307.

Gould, S.J., 1977. Ontogeny and Phylogeny. Harvard University Press, Cambridge.

Guy, F., Lieberman, D.E., Pilbeam, D., Ponce de León, M., Likius, A., Mackaye, H.T., Vignaud, P., Zollikofer, C., Brunet, M., 2005. Morphological affinities of the Sahelanthropus tchadensis (late Miocene hominid from Chad) cranium. Proc. Natl. Acad. Sci. 102, 18836-18841.

Hallgrimsson, B., Lieberman, D., Liu, W., Ford-Hutchinson, A.F., Jirik, F.R., 2007. Epigenetic interactions and the structure of phenotypic variation in the cranium. Evol. Dev. 9, 76-91.

Heintz, N., 1966. Le crâne des anthropomorphes: croissance relative, variabilité, evolution. Musée Royal de L'Afrique Centrale-Tervuren. Annales Science Zoologiques (n.s.) 6, 1-122.

Jungers, W.L., Falsetti, A.B., Wall, C.E., 1995. Shape, relative size, and size adjustments in morphometrics. Yearb. Phys. Anthropol. 38, 137-161.

Kelley, J., Smith, T.M., 2003. Age at first molar emergence in early Miocene Afropithecus turkanensis and life-history evolution in the Hominoidea. J. Hum. Evol. 44, 307-329.

Kinzey, W.G., 1984. The dentition of the pygmy chimpanzee, Pan paniscus. In: Susman, R.L. (Ed.), The Pygmy Chimpanzee: Evolutionary Biology and Behavior. Plenum Press, New York, pp. 65-88.

Klingenberg, C.P., 1998. Heterochrony and allometry: the analysis of evolutionary change in ontogeny. Biol. Rev. Camb. Philos. Soc. 73, 79-123. 
Kuykendall, K.L., 1996. Dental development in chimpanzees (Pan troglodytes): the timing of tooth calcification stages. Am. J. Phys. Anthropol. 99, 135-157.

Leigh, S.R., Shah, N.F., Buchanan, L.S., 2003. Ontogeny and phylogeny in papionin primates. J. Hum. Evol. 45, 285-316.

Lieberman, D.E., 1996. How and why recent humans grow thin skulls: experimental data on systemic cortical robusticity. Am. J. Phys. Anthropol. 101, 217-236.

Lieberman, D.E., Mowbray, K.M., Pearson, O.M., 2000a. Basicranial influences on overall cranial shape. J. Hum. Evol. 38, 291-315.

Lieberman, D.E., Ross, C.F., Ravosa, M.J., 2000b. The primate cranial base: Ontogeny, function, and integration. Yearb. Phys. Anthropol. 43, $117-169$.

Lieberman, D.E., Krovitz, G.E., McBratney-Owen, B., 2004. Testing hypotheses about tinkering in the fossil record: the case of the human skull. J. Exp. Zool. (Mol. Dev. Evol.) 302B, 284-301.

McHenry, M., Corruccini, R.S., 1981. Pan paniscus and human evolution. J. Hum. Evol. 54, 355-367.

McKinney, M.L., McNamara, K.J., 1991. Heterochrony: The Evolution of Ontogeny. Plenum Press, New York.

Mitteroecker, P., Gunz, P., Bernhard, M., Schaefer, K., Bookstein, F.L., 2004. Comparison of cranial ontogenetic trajectories among great apes and humans. J. Hum. Evol. 46, 679-698.

Mitteroecker, P., Gunz, P., Bookstein, F.L., 2005. Heterochrony and geometric morphometrics: a comparison of cranial growth in Pan paniscus versus Pan troglodytes. Evol. Dev. 7, 244-258.

Moss, M.L., 1958. The pathogenesis of artificial cranial deformation. Am. J. Phys. Anthropol. 6, 269-286.

Moss, M.L., Young, R.W., 1960. A functional approach to craniology. Am. J. Phys. Anthropol. 18, 281-292.

O'Higgins, P., 2000. Quantitative approaches to the study of craniofacial growth and evolution: advances in morphometric techniques. In: O'Higgins, P., Cohn, M. (Eds.), Development, Growth and Evolution: Implications for the Study of Hominid Evolution. Academic Press, London, pp. 163-185.

Penin, X., Berge, C., Baylac, M., 2002. Ontogenetic study of the skull in modern humans and the common chimpanzees: neotenic hypothesis reconsidered with a tridimensional Procrustes analysis. Am. J. Phys. Anthropol. $118,50-62$.

Ponce de León, M.S., Zollikofer, C.P.E., 2006. Neanderthals and modern humans-chimps and bonobos: Similarities and differences in development and evolution. In: Harvati, K., Harrison, T. (Eds.), Neanderthals Revisited: New Approaches and Perspectives. Springer, New York, pp. 71-90.

Rice, S.H., 1997. The analysis of ontogenetic trajectories: When a change in size or shape is not heterochrony. Proc. Nat. Acad. Sci. 94, 907-912.

Rohlf, F.J., Slice, D.E., 1990. Extensions of the Procrustes methods for the optimal superimposition of landmarks. Syst. Zool. 39, 40-59.

Schultz, A.H., 1960. Age changes in primates and their modification in man. In: Tanner, J.M. (Ed.), Human Growth. Pergamon, Oxford, pp. 1-20.

Schwarz, E., 1929. Das vorkommen des Schimpanzen auf den linked KongoUfer. Rev. Zool. Bot. Afr. 16, 425-426.

Shea, B.T., 1983a. Paedomorphosis and neoteny in the pygmy chimpanzee. Science 222, 521-522.
Shea, B.T., 1983b. Allometry and heterochrony in the African apes. Am. J. Phys. Anthropol. 62, 275-289.

Shea, B.T., 1984. An allometric perspective on the morphological and evolutionary relationships between pygmy (Pan paniscus) and common (Pan troglodytes) chimpanzees. In: Susman, R.L. (Ed.), The Pygmy Chimpanzee: Evolutionary Biology and Behavior. Plenum Press, New York, pp. 175-205.

Shea, B.T., 1985. Ontogenetic allometry and scaling: A discussion based on the growth and form of the skull in African apes. In: Jungers, W.L. (Ed.), Size and Scaling in Primate Biology. Plenum Press, New York, pp. 175-205.

Shea, B.T., 1989. Heterochrony in human evolution: the case for neoteny reconsidered. Yearb. Phys. Anthropol. 32, 69-104.

Shea, B.T., 1992. Neoteny. In: Jones, S., Martin, R., Pilbeam, D. (Eds.), The Cambridge Encyclopedia of Human Evolution. Cambridge University Press, Cambridge, pp. 401-404.

Shea, B.T., 2000. Current issues in the investigation of evolution by heterochrony, with emphasis on the debate over human neoteny. In: Parker, S., Langer, J., McKinney, M.L. (Eds.), The Evolution of Behavioral Ontogeny. School of American Research Press, Santa Fe, pp. 181-214.

Smith, B.H., 1989. Dental development as a measure of life history among primates. Evolution 43, 683-688.

Smith, B.H., 1991. Dental development and the evolution of life history in Hominidae. Am. J. Phys. Anthropol. 86, 157-174.

Smith, B.H., Crummet, T.L., Brandt, K.L., 1994. Ages of eruption of primate teeth: a compendium for aging individuals and comparing life histories. Yearb. Phys. Anthropol. 37, 177-231.

Smith, K.K., 2001. Heterochrony revisited: the evolution of developmental sequences. Biol. J. Linn. Soc. 73, 169-186.

Smith, R.J., Leigh, S.R., 1998. Sexual dimorphism in primate neonatal body mass. J. Hum. Evol. 34, 173-201.

Tissot, B.N., 1988. Multivariate analysis. In: McKinney, M.L. (Ed.), Heterochrony in Evolution: A Multidisciplinary Approach. Plenum Press, New York, pp. 35-51.

Weidenreich, F., 1941. The extremity bones of Sinanthropus pekinensis. Palaeontol. Sin. New Ser. D 5, 1-150.

Williams, F.L., Godfrey, L.R., Sutherland, M.R., 2002. Diagnosing heterochronic perturbations in the craniofacial evolution of Homo (Neanderthals and modern humans) and Pan ( $P$. troglodytes and $P$. paniscus). In: Thompson, J.L., Krovitz, G., Nelson, A.J. (Eds.), Patterns of Growth and Development in the Genus Homo. Cambridge University Press, Cambridge, pp. $405-441$.

Zelditch, M.L., Fink, W.L., 1996. Heterochrony and heterotopy: stability and innovation in the evolution of form. Paleobiology 22, 241-254.

Zelditch, M.L., Fink, W.L., Swiderski, D.L., 1995. Morphometrics, homology, and phylogenetics: Quantified characters as synapomorphies. Syst. Biol. 44, 179-189.

Zelditch, M.L., Swiderski, D., Sheets, D., Fink, W.L., 2004. Geometric Morphometrics for Biologists: A Primer. Elsevier, London.

Zihlman, A., Bolter, D., Boesch, C., 2004. Wild chimpanzee dentition and its implications for assessing life history in immature hominin fossils. Proc. Natl. Acad. Sci. 101, 10541-10543.

Zollikofer, C.P.E., Ponce de León, M.S., 2004. Kinematics of cranial ontogenesis: Heterotopy, heterochrony, and geometric morphometric analysis of growth models. J. Exp. Zool. (Mol. Dev. Evol.) 302B, 322-340. 